\title{
Facial Diplegia in Neuroinvasive West Nile Virus Infection
}

\author{
Tommy L.H. Chan, Adrian Budhram
}

Keywords: West Nile virus, Acute flaccid paralysis, Facial diplegia

doi:10.1017/cjn.2019.46

Can J Neurol Sci. 2019; 46: 453-454

An 81-year-old woman presented with facial weakness preceded by a 2 -week flu-like illness. Her past medical history included type 1 diabetes and Crohn's disease. Neurologic examination revealed facial diplegia in a lower motor neuron distribution, with no other cranial nerve, motor, sensory or coordination deficit (Figure 1). General examination did not demonstrate any cardiorespiratory, gastrointestinal or rheumatological findings. No rash was detected. Brain magnetic resonance imaging (MRI) revealed subtle signal abnormality in the dorsal pons without restricted diffusion (Figure 2). There were findings compatible with a bilateral facial motor axonal neuropathy on nerve conduction studies (NCS); both blink reflexes were absent when bilateral supraorbital nerves were stimulated and recorded over the orbicularis oculi, and bilateral facial motor responses from the nasalis were low in amplitude. Cerebrospinal fluid (CSF) profile

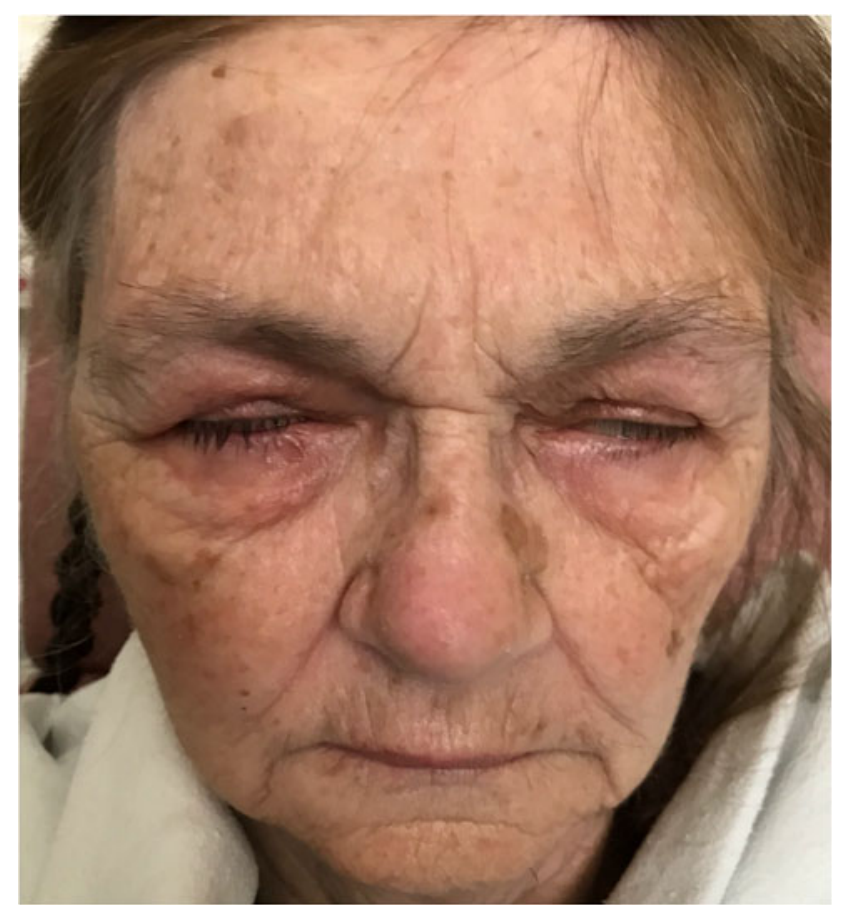

Figure 1: Facial diplegia in neuroinvasive WNV infection. On attempted simultaneous eyelid closure and facial grimace, incomplete eye closure and absent activation of the musculature of the lower face was seen. demonstrated a neutrophilic-predominant pleocytosis with 120 nucleated cells. Gram stain and culture as well as viral polymerase chain reaction tests for enterovirus, herpes simplex virus and varicella zoster virus were negative in CSF. Serum infectious workup was negative for human immunodeficiency virus, syphilis, hepatitis B, C and Lyme disease. Serum angiotensin-converting enzyme was within normal limits and chest X-ray showed no evidence of sarcoidosis. West Nile virus (WNV) IgM was reactive in both CSF and serum, compatible with acute WNV infection. Unfortunately, she had a complicated course in hospital. Her oral intake was significantly impaired from her facial diplegia and she developed hospital-acquired delirium. Speech language pathologist assessment identified oral stage dysphagia with anterior leakage related to her bilateral facial diplegia as well as an up and down mashing mastication pattern difficulty with bolus transport and lingual thrusting. A gastrojejunostomy tube was placed, but she later developed an upper respiratory tract infection and worsening delirium. Ultimately, she was palliated and the primary goal was comfort-focused care.

Acute flaccid paralysis (AFP) is a classic neuromuscular manifestation of WNV and typically presents as limb weakness. Approximately $11-17 \%$ of patients with neuroinvasive WNV may demonstrate facial paralysis; therefore, neuroinvasive WNV infection should be included in the differential diagnosis of acquired facial diplegia. ${ }^{1,2}$ Other causes of facial diplegia include Guillain Barre Syndrome, Myasthenia Gravis, neoplasm, syphilis, human immunodeficiency virus, Lyme disease, sarcoidosis and systemic lupus erythematosus. ${ }^{3}$ Testing for CSF WNV IgM is the gold standard for diagnosis in patients with suspected neuroinvasive WNV infection. The CSF usually demonstrates elevated protein and mild-to-moderate pleocytosis with a predominance of lymphocytes. However, neutrophils may predominate in early infection. ${ }^{4}$ Neuroimaging may demonstrate signal abnormalities depending on the areas of involvement, and electrodiagnostic studies can support a motor neuronopathy in patients with AFP. Treatment for neuroinvasive WNV infection remains primarily supportive, with little

From the Department of Clinical Neurological Sciences, Western University, London, Ontario, Canada

Received January 4, 2019. Final Revisions Submitted April 2, 2019. Date of ACCEPTANCE April 3, 2019.

Correspondence to: Tommy L.H. Chan, Department of Clinical Neurological Sciences, London Health Sciences Centre (LHSC), University of Western Ontario, London, Ontario, Canada N6A 5A5. Email: tommy.chan@lhsc.on.ca 


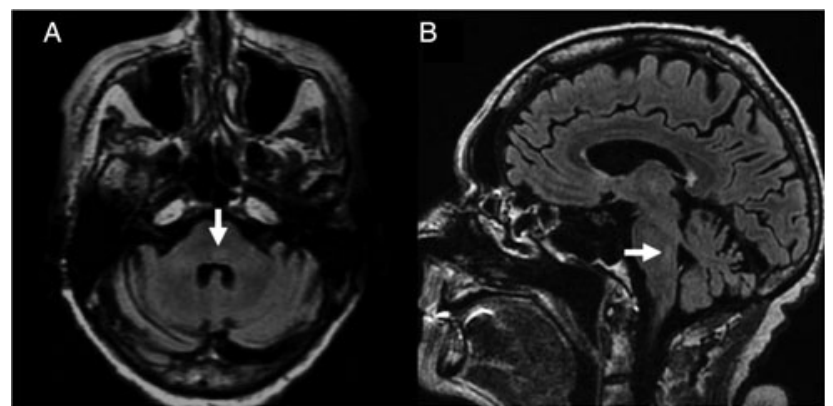

Figure 2: Brain MRI in neuroinvasive WNV infection. On axial (A) and sagittal (B) fluid-attenuated inversion recovery imaging, T2 hyperintensity of the dorsal pons is seen (arrows).

evidence to support the use of therapies such as interferon, ribavirin and intravenous immunoglobulin. ${ }^{5}$ Eye care is vital in the context of facial paralysis to avoid corneal abrasions in patients with incomplete eye closure. The prognosis for neuroinvasive WNV varies, with old age, immunosuppression, altered level of consciousness and respiratory involvement carrying a higher risk of mortality. ${ }^{4}$

\section{Statement of Authorship}

TLHC examined the patient. Both authors wrote and revised the manuscript and created the figures together.

\section{Disclosures}

The authors have no conflicts of interest to declare. "Patient Consent for Publication" form was signed and obtained from the patient.

\section{REFERENCE}

1. Jeha LE, Sila CA, Lederman RJ, Prayson RA, Isada CM, Gordon SM. West Nile virus infection: a new acute paralytic illness. Neurology. 2003;61(1):55-9.

2. Pepperell C, Rau N, Krajden S, et al. West Nile virus infection in 2002: morbidity and mortality among patients admitted to hospital in southcentral Ontario. CMAJ. 2003;168(11):1399-405.

3. Keane JR. Bilateral seventh nerve palsy: analysis of 43 cases and review of the literature. Neurology. 1994;44(7):1198-202.

4. Sejvar JJ, Bode AV, Marfin AA, et al. West Nile virus-associated flaccid paralysis. Emerging Infect Dis. 2005;11(7):1021-7.

5. Planitzer CB, Modrof J, Kreil TR. West Nile virus neutralization by US plasma-derived immunoglobulin products. J Infect Dis. 2007;196(3):435-40. 